\title{
EL CONOCIMIENTO, CONCEPTOS VALORATIVOS Y LA PRESERVACIÓN DEL MEDIO AMBIENTE
}

\author{
KNOWLEDGE, VALUABLE CONCEPTS AND THE PRESERVATION OF THE \\ ENVIRONMENT \\ Juilo Cesar Trujwo Meza* \\ Docente Principal de la Facultad de Ciencias Contables \\ Universidad Nacional Mayor de San Marcos-UNMSM / Lima-Perú \\ Morena Colorado de Hernández ${ }^{* *}$ \\ Docente en la Universidad de El Salvador \\ [Recepción: Agosto 2016/ Conformidad: Setiembre 2016]
}

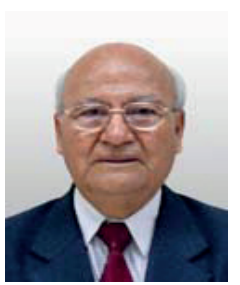

\section{RESUMEN}

La asistencia al evento programado por la Alafec y la Universidad de Texas Rio Grande Valley, Bronwsville fue por interés particular de seguir con las movilidades internacionales; la primera fue en la Republica de Panamá sobre el razonamiento bioético aplicado a las empresas socialmente responsables; y en esta segunda oportunidad fue validar e investigar una metodología de investigación utilizando el programa de SPSS para los procedimientos estadísticos y conocer el proceso científico para realizar investigaciones empíricas y análisis que corresponde para llevar a los resultados de dicha investigación.

La investigación se inició con el estudio de una serie de muestras donde se considera las variables, dependientes como las independientes y se sometió al análisis de 758 muestras y se consideraron los conceptos relacionados con: El consumo responsable; el comercio justo; la soberanía alimenticia; el desarrollo sostenible y sustentable de modo que se contribuya a preservar el medio ambiente a nivel de Latinoamérica.

\section{Palabras Clave:}

razonamiento bioético, comercio justo, consumo responsable.

\section{ABSTRACT}

Attendance at the event programmed by Alafec and the University of Texas Rio Grande Valley- Bronwsville, was for the particular interest of continuing international mobilizations. The first, was in the Republic of Panama on the bioethical reasoning applied to socially responsible companies; and this second opportunity was to validate and investigate a research methodology using the SPSS program for statistical procedures and to know the scientific process to carry out empirical research and the analysis corresponding to lead the results of such research.

The investigation began with a series of study samples where the variables -dependent and independentwere considered. Were submitted 758 samples to the analysis and the concepts related to: Responsible consumption; Fair trade; Food sovereignty; Sustainable development and the contribution to the preservation of the environment in Latin America.

\section{Keywords:}

Bioethical Reasoning, Fair Trade, Responsible Consumption.

\footnotetext{
* Magister, Docente Principal en la UNMSM. Email: estudiotrujillo@yahoo.com

** Docente en la Universidad de El Salvador. Email: morena.colorado@ues.edu.sv
} 


\section{INTRODUCCIÓN}

En la actualidad el mundo atraviesa por una crisis ambiental y coyuntural en cuanto al Modelo de Economía impuesta por los Países Desarrolladas, modelo o sistema de la globalización. La globalización fue impuesta aproximadamente los años 1980 cuando las grandes potencias lideradas por Estados Unidos y Gran Bretaña vencieron en la Guerra económica librada entre Países Capitalistas y Países Socialistas, ganando los países capitalistas e imponiendo el Sistema de la Globalización.

No obstante lo antes indicado permanece en el mundo tres sistemas o modelos de economía que marca las conductas sociales y políticas sobre todo de los países emergentes o subdesarrollados, ellos pueden alinearse a cualquiera de los Sistemas económicos. El Capitalismo lo lidera Estados Unidos caracterizándose el predominio de la propiedad privada y de las libertades sin límites; el Modelo de la Social Democracia, se sustenta en un Sistema donde coexisten la propiedad privada y la publica en el 50\% para cada una'. Un sistema aparentemente más justo y tiene una duración aproximada de 40 años de vigencia desde el término de la segunda Guerra mundial, Sistema liderado por Alemania y; finalmente, el Sistema Socialista donde se desarrolla una economía dirigida, en el $100 \%$, por el Estado quien tiene todos los medios de producción, si bien los países emergentes pueden adherirse a cualquiera de los tres sistemas, en la práctica se ha impuesto el Sistema de la Globalización imperante en el mundo salvo algunos países que combinan ambos sistemas o tratan de crear nuevos modelos económicos.

En el transcurrir del tiempo se impuso la ideología del libre Mercado porque en la práctica, a través del Fondo Monetario Internacional (FMI) y el Banco Mundial (BM), impusieron dicho modelo a los países emergentes debido al requerimiento de capital, préstamos y subvenciones. Joseph Stiglitz, premio nobel de economía en el año 2008 de nacionalidad Estadounidense, en su obra: "El Precio de la Igualdad" no está de acuerdo con el Sistema y decía que debían de aplicarse muchos ajustes al Sistema, decía que en las transacciones económicas de nuestros productos primarios debía haber un sinceramiento y no seguir pagando las materias primas por debajo del costo del producto y con fuertes márgenes de ganancia.
En Latinoamérica desde entonces el modelo de la globalización ha traído más pobreza y el incremento de la desigualdad conforme afirma Thomas Piketty, economista Ingles quien en el año 2015 publico su libro: "La Economía del Siglo XXI", señalándo en sus estudios su discrepancia en cuanto al Sistema Global, indicando que en el mundo actual, el 1\% de la población poseé tanta riqueza o más, que el $99 \%$ restante. Recomendando, entre otras cosas, una reforma de la economía de la Globalización, un cambio del Sistema Tributario para que paguen más impuestos los que más tienen, cumpliendo el principio de la "capacidad contributiva" y sugiere reformas en los Sistemas Tributarios en el mundo, respetando los principios obligatorios y no obligatorios en materia económica y tributaria.

La situación planteada nos hace ver que se trata de un problema económico para el Capitalismo; pero para los países emergentes se trata de un problema ético en principio, al no respetar los cuatro principios de la bioética ni de los principios antes indicados; en la medida que, dicha postura afecta a la vida de las personas, habiendo llegado en la actualidad, a una complicación del medio ambiente, con ello acelerar la crisis en todo orden y hasta la posibilidad de extinción del planeta.

De ello resulta un consenso de países emergentes sobre la necesidad de cambio de políticas de crecimiento sostenible para lograr un desarrollo sostenible relacionado con el medio ambiente, con lo social, con lo económico y lo político, pero el desarrollo sostenible debe tener su base en el: comercio justo, el consumo responsable y la soberanía alimentaria, vistos como fenómenos sociales que son criterios y conceptos a ser analizados en el presente trabajo de investigación; sin embargo, considero debe ser una variable dependiente con sus variables independientes para alcanzar dichos objetivos.

\section{OBJETIVO Y ALCANCE DEL TRABAJO}

El objetivo del estudio es, la aplicación de una investigación metodológica que abarque la investigación cualitativa y cuantitativa. Para ello, debe tener en cuenta lo siguiente:

1. El objetivo del estudio es la aplicación de una

140/ QVIPUKAMAYOC | Vol. 24(46) 2016 
metodología de la investigación, a las encuestas previamente programadas y realizadas a los estudiantes de pre y Posgrado de varios Países Latinoamericanos, para observar sus actitudes y conductas, teniendo en cuenta el conocimiento y utilización de la Bioética y la Ética, aplicada al comportamiento de los estudiantes frente a los conceptos: de la Bioética, la Ética, el desarrollo sostenible, el Consumo Responsable, El Comercio Justo, la Soberanía Alimentaria, La Inquietud Ética y la Virtud Ética, conceptos que en su aplicación tienen que ver con situaciones de sobrevivencia frente al ecosistema y el cuidado del medio ambiente.

2. A efectos de investigar lo señalado, procedimos analizar el Marco Teórico de los diferentes conceptos indicados en el párrafo anterior y otros que sean aplicables en las circunstancias, a fin de enfocar la investigación a buscar respuestas de cambio de actitudes y conductas de las personas. Para determinar conclusiones aplicables en las circunstancias; utilizando para ello, una metodología que tenga en cuenta autores, actuales y anteriores, que escribieron al respecto; así como también, la metodología especial de las profesoras: Silvia López Palau y Beatriz Rivera Cruz, tanto en sus estudios del 2012 y los que proponen ahora.

3. En el proceso de la investigación, empleamos una metodología científica, con la intervención de la estadística del Sistema SPSS, aplicaremos las teorías conductuales de: Modelo de Toma de Decisiones Éticas; Modelo Transteórico de etapas de cambio; Teoría de la Disonancia Cognitiva y fundamentalmente, el Estudio del Razonamiento Bioético, adaptadas a las variables dependientes e independientes, a fin de obtener los resultados o tendencias que puedan ser utilizadas como conclusiones válidas y empleadas como resultado de la Investigación, y si fuera el caso, plantear recomendaciones.

\section{MARCO TEÓRICO CONCEPTUAL:}

El marco teórico conceptual del tema propuesto es bastante amplio; sin embargo, abordaremos lo referido al objeto del estudio teniendo en cuenta lo siguiente:
El alcance de la Bioética como elemento fundamental, en la medida de la aplicación de los principios de: Autonomía, la no Maleficencia, la Beneficencia y la Justicia, que son principios necesarios y primordiales, en la valoración y aplicación del alcance de la Ética en sus diversas manifestaciones.

El Desarrollo Sostenible, aunado al mecanismo de satisfacción de las necesidades de la generación presente, sin comprometer la capacidad de generación futura; $y$ en esta forma, satisfacer las necesidades de la Humanidad.

El Comercio Justo, concepto que nos lleva al empleo de actitudes y conductas éticas en el comportamiento de las personas de los países desarrollados, así como la de los emergentes; y sobre todo, en el comercio internacional, a fin de hacer comprender y buscar nuevas alternativas frente a la ideología capitalista, para que en el comercio interno y externo se determinen los costos más justos y se tenga en cuenta un margen razonable y justo de utilidad sin disminuir la calidad. Esto requiere una profunda reflexión para beneficio de las mujeres y niños; así como, de los más necesitados y en esta forma mantener un consumo responsable que preserve el medio ambiente, que es el objetivo del análisis y utilización de los conceptos auto analizados.

La Soberanía Alimentaria, concepto que preocupa al mundo, basta observar que en 1996 se realiza una Cumbre Mundial donde se analiza la situación de los pueblos y países con mayores necesidades alimentarias, recomendando políticas agrícolas, laborales, de pesca y otras, de acuerdo a sus circunstancias particulares, de modo que puedan preservar el medio ambiente y el consumo responsable, impulsando un crecimiento armónico y un desarrollo sostenido.

La Ética de la Virtud vinculada con la aplicación de la bioética y la ética en sí, valorando los alcances de la deontología y la teología en sus diferentes manifestaciones, de modo que se cambie de actitud y conducta frente a la observancia de ciertos principios que contribuyan la sostenibilidad del crecimiento económico de los países y del correspondiente desarrollo.

Las teorías Conductuales en la toma de decisiones éticas; así como, el Modelo Transteórico de 
Etapas de Cambio, como posiciones y antecedentes necesarios en el estudio y en la aplicación a la metodología de investigación propuesta.

Consideramos que el modelo que se adapta a la investigación, presenta una metodología que propone un instrumento de medición desarrollado y propuesto por las Doctoras Silvia López Palau y Beatriz Rivera Cruz (2026-b), con una propuesta Latinoamericana.

El Modelo antes indicado está integrado por los modelos de Rest (1986), la teoría de la Disonancia Cognitiva de Festinger (1957) y el modelo Transteórico de Etapas de Cambio de Prochaska \& Diclemente (1984).

Estos son los conceptos principales a tenerse en cuenta en el proceso de investigación y que fueron utilizados en el presente trabajo, a parte de otros conceptos vinculados a estos estudios, con el objetivo de lograrse un desarrollo sostenible y contribuir en forma plena, a la conservación del medio ambiente.

\section{METODOLOGÍA}

El proceso de la metodología de la investigación científica pretende señalar un diseño o modelo de investigación, con la aplicación del método estadístico del SPSS y en contraste con una muestra Latinoamericana, teniendo como base fundamental los conceptos siguientes:

a) El conocimiento de la base teórica conceptual, teniendo en cuenta La Responsabilidad Social Empresarial en su extensión al propósito de la presente investigación; Los principios Éticos, la inquietud ética, la virtud ética, el comercio justo; el consume responsable; el desarrollo sostenible y las soberanía alimentaria; y otras aplicables a dicha metodología, para lograr el desarrollo sostenible. b) El empleo del instrumento del Sistema SPSS, mecanismo estadístico, aplicado a los RESULTADOS: efectos de la cuantificación y las tendencias, como respuesta del análisis de las variables dependientes e independientes, originados por la muestra Latinoamericana, por la utilización de los procedimientos inherentes al Sistema propuesto.

c) Introducción de la base de datos de acuerdo con la muestra Latinoamericana, presentada como parte del modelo metodológico, su análisis, resultados, la evaluación y la cuantificación de sus resultados, para llegar a sus conclusiones y si fuera el caso recomendaciones.

\section{COMPOSICIÓN DE LA MUESTRA}

En razón de la muestra sometida al análisis estadístico y demás, paso a exponer los resultados de cada concepto evaluado estadísticamente. La muestra total consiste de 758 estudiantes universitarios (pregrado y posgrado); alrededor del 95\% de la muestra, pertenece a la generación de los mileniales y alrededor de dos terceras partes de los participantes, están considerados que tienen un poder adquisitivo de medio a bajo.

\section{RESULTADOS}

\section{Indice de conocimiento del Desarrollo Sostenible}

El nivel de conocimiento de los participantes sobre los temas tratados es bastante limitada. Menos de la mitad de las premisas obtuvieron promedios mayores de 50\%. Las premisas con los promedios más altos, aunque no llegan al 70\%, están relacionados al consumo responsable. Los participantes parecen tener cierto conocimiento sobre el consumo responsable y algunos aspectos del desarrollo sostenible. (Ver Tabla $\mathrm{N}^{\circ} 01$ ) 
Tabla No 01:

Indice de Conocimiento Sobre Desarrollo Sostenible (Objetivo $\mathrm{N}^{\circ} 1$ )

\begin{tabular}{|c|c|c|}
\hline \multirow{2}{*}{ PREMISAS } & \multicolumn{2}{|c|}{ CONOCIMIENTO } \\
\cline { 2 - 3 } & Promedio & Desv.Est. \\
\hline
\end{tabular}

\section{COMERCIO JUSTO}

Estrategia para aliviar la pobreza y propiciar el desarrollo sostenible

Promueve activamente mejores prácticas medioambientales

Permite una produccion socialmente justa y responsable

Valora y recompensa debidamente el trabajo de la mujer

Da oportunidad a productores en desventaja

Se mantienen relaciones basadas en la solidaridad y la confianza

Los prodcutores trabajan un entorno seguro y saludable

Utilizan técnicas honestas de publicidad y mercadeo

Siguen estándares más altos en calidad y empaquetado del producto

El precio justo se acuerda a través del diálogo y la participación

La participación de niños no afecta adversamente su bienestar

Las organizaciones de CJ generan conciencia sobre CJ

Indíces de conocimiento e inquietud Comercio Justo

\begin{tabular}{|l|l|}
\hline 51.0 & 27.0 \\
\hline 48.9 & 30.5 \\
\hline 49.5 & 29.6 \\
\hline 48.1 & 31.1 \\
\hline 49.3 & 29.1 \\
\hline 46.8 & 30.6 \\
\hline 47.1 & 30.1 \\
\hline 45.1 & 30.1 \\
\hline 46.6 & 30.0 \\
\hline 46.2 & 30.9 \\
\hline 40.3 & 31.6 \\
\hline 37.5 & 29.9 \\
\hline 46.3 & $\mathbf{2 2 . 0}$ \\
\hline
\end{tabular}

\section{CONSUMO RESPONSABLE}

\begin{tabular}{|l|l|l|}
\hline Distinguen entre sus necesidades y los deseos & 66.9 & 27.6 \\
\hline Esta motivado por su postura ética & 61.8 & 29.8 \\
\hline Mantiene su compromiso con el medioambiente & 63.3 & 28.7 \\
\hline Minimiza el consumo y evita el despilfarro & 67.4 & 28.4 \\
\hline Esta interesado por el origen del producto y a quién beneficia & 58.7 & 29.8 \\
\hline Paga un sobreprecio por la misma calidad si hay un valor ético & 56.6 & 30.0 \\
\hline Consideran dónde y cómo se ha fabricado el producto & 54.4 & 32.3 \\
\hline Indíces de conocimiento e inquietud Consumo Responsable & $\mathbf{6 1 . 4}$ & $\mathbf{2 2 . 1}$ \\
\hline
\end{tabular}

DESARROLLO SOSTENIBLE

\begin{tabular}{|l|c|c|}
\hline Los problemas ambientales de Latinoamérica & 61.8 & 30.4 \\
\hline Los bosques tropicales han perdido 80\% de su superficie & 59.0 & 30.8 \\
\hline Definición de desarrollo sostenible & 59.9 & 31.4 \\
\hline Los transgénicos y el glifosato causan daños a la salud & 53.5 & 33.4 \\
\hline 76 países han perdido ya todos sus bosques primarios & 48.0 & 31.3 \\
\hline Indíces de conocimiento e inquietud Desarrollo Sostenible & $\mathbf{5 6 . 4}$ & $\mathbf{2 4 . 0}$ \\
\hline \multicolumn{1}{|c|}{ SOBERANÍA ALIMENTARIA } & 51.8 & 29.8 \\
\hline Producción de alimentos se basa en la sostenibilidad & 52.7 & 32.3 \\
\hline Definición de soberanía alimentaria & 50.9 & 31.5 \\
\hline Respeta el derecho de alimentación y nutrición de los consumidores & 50.7 & 31.6 \\
\hline Da prioridad a las economías y a los mercados locales y nacionales & 49.4 & 30.9 \\
\hline Promueve comercio transparente que garantiza ingresos dignos & 46.0 & 31.4 \\
\hline Se opone a la mercantilización y las patentes de los bienes comunes & 50.5 & 26.0 \\
\hline Indíces de conocimiento e inquietud soberanía alimentaria & $\mathbf{5 4 . 7}$ & $\mathbf{1 8 . 3}$ \\
\hline INDÍCE DE CONOCIMIENTO E INQUIETUD GENERAL & & 2 \\
\hline
\end{tabular}

Fuente: Elaboración propia. 


\section{Indice de Inquietud sobre el Desarrollo Sostenible}

En términos del índice de inquietud ética, la mayoría de las premisas obtuvieron valores sobre el $70 \%$ y todas están relacionadas al desarrollo sostenible, siendo al parecer la preocupación principal de los participantes sobre los temas expuestos.

(Ver Tabla $\mathrm{N}^{\circ}$ 02)

Tabla No 02:

Indice de Inquietud Ética Sobre Desarrollo Sostenible (Objetivo $\mathrm{N}^{\circ} 2$ )

\begin{tabular}{|c|c|c|}
\hline \multirow{2}{*}{ PREMISAS } & \multicolumn{2}{|c|}{ INQUIETUD ÉTICA } \\
\cline { 2 - 3 } & Promedio & Desv.Est. \\
\hline
\end{tabular}

\section{COMERCIO JUSTO}

\begin{tabular}{|l|l|l|}
\hline Estrategia para aliviar la pobreza y propiciar el desarrollo sostenible & 64.4 & 30.4 \\
\hline Promueve activamente mejores prácticas medioambientales & 69.1 & 31.3 \\
\hline Permite una produccion socialmente justa y responsable & 64.8 & 30.8 \\
\hline Valora y recompensa debidamente el trabajo de la mujer & 71.5 & 31.2 \\
\hline Da oportunidad a productores en desventaja & 61.2 & 29.0 \\
\hline Se mantienen relaciones basadas en la solidaridad y la confianza & 65.5 & 32.2 \\
\hline Los prodcutores trabajan un entorno seguro y saludable & 65.5 & 31.0 \\
\hline Utilizan técnicas honestas de publicidad y mercadeo & 60.1 & 31.1 \\
\hline Siguen estándares más altos en calidad y empaquetado del producto & 62.3 & 30.8 \\
\hline El precio justo se acuerda a través del diálogo y la participación & 64.1 & 30.6 \\
\hline La participación de niños no afecta adversamente su bienestar & 70.9 & 30.9 \\
\hline Las organizaciones de CJ generan conciencia sobre CJ & 54.3 & 31.9 \\
\hline Indíces de conocimiento e inquietud Comercio Justo & $\mathbf{6 4 . 5}$ & $\mathbf{2 4 . 4}$ \\
\hline
\end{tabular}

CONSUMO RESPONSABLE

\begin{tabular}{|l|l|l|}
\hline Distinguen entre sus necesidades y los deseos & 67.6 & 31.5 \\
\hline Esta motivado por su postura ética & 64.8 & 32.3 \\
\hline Mantiene su compromiso con el medioambiente & 70.9 & 30.7 \\
\hline Minimiza el consumo y evita el despilfarro & 68.8 & 31.7 \\
\hline Esta interesado por el origen del producto y a quién beneficia & 65.4 & 31.2 \\
\hline Paga un sobreprecio por la misma calidad si hay un valor ético & 67.6 & 30.5 \\
\hline Consideran dónde y cómo se ha fabricado el producto & 67.9 & 32.4 \\
\hline Indíces de conocimiento e inquietud Consumo Responsable & $\mathbf{6 7 . 0}$ & $\mathbf{2 5 . 4}$ \\
\hline
\end{tabular}

DESARROLLO SOSTENIBLE

\begin{tabular}{|l|c|c|}
\hline Los problemas ambientales de Latinoamérica & 81.8 & 24.9 \\
\hline Los bosques tropicales han perdido 80\% de su superficie & 80.8 & 25.5 \\
\hline Definición de desarrollo sostenible & 72.4 & 29.8 \\
\hline Los transgénicos y el glifosato causan daños a la salud & 76.1 & 27.9 \\
\hline 76 países han perdido ya todos sus bosques primarios & 80.8 & 25.5 \\
\hline Indíces de conocimiento e inquietud Desarrollo Sostenible & $\mathbf{7 8 . 4}$ & $\mathbf{2 2 . 6}$ \\
\hline \multicolumn{2}{|c|}{ SOBERANÍA ALIMENTARIA } & \multicolumn{2}{|c|}{} \\
\hline Producción dealimentos se basa en la sostenibilidad & 68.7 & 30.7 \\
\hline Definición de soberanía alimentaria & 70.9 & 30.6 \\
\hline Respeta el derecho de alimentación y nutrición de los consumidores & 69.0 & 31.7 \\
\hline Da prioridad a las economías y a los mercados locales y nacionales & 70.1 & 30.9 \\
\hline Promueve comercio transparente que garantiza ingresos dignos & 70.8 & 29.8 \\
\hline Se opone a la mercantilización y las patentes de los bienes comunes & 69.1 & 29.7 \\
\hline Indíces de conocimiento e inquietud soberanía alimentaria & 69.7 & 26.3 \\
\hline INDÍCE DE CONOCIMIENTO E INQUIETUD GENERAL & $\mathbf{6 9 . 9}$ & $\mathbf{2 0 . 5}$ \\
\hline
\end{tabular}

Fuente: Elaboración propia.

144/ QVIPURAMAYOC | Vol. 24(46) 2016 


\section{Diferencias entre el conocimiento y la inquietud ética}

Cuatro premisas del desarrollo sostenible y una de la soberanía alimentaria y una de comercio justo obtuvieron las diferencias más altas y significativas entre el nivel de conocimiento y el de inquietud ética. En todas las premisas.

Los participantes tienen más inquietud que cono- cimiento sobre el tema. En las que no hay diferencias significativas entre el nivel de conocimiento y la inquietud ética están relacionadas mayoritariamente al consumo responsable, que se encuentran entre las que más conocen los participantes, y del comercio justo. (Ver Tabla $\mathrm{N}^{\circ} 03$ )

\section{Tabla No 03:}

Diferencias entre el nivel de conocimiento y la inquietud ética (Objetivo $\mathrm{N}^{\circ} 3$ )

\begin{tabular}{|c|c|c|}
\hline PREMISAS & $\begin{array}{l}\text { DIFERENCIA DE } \\
\text { LOS PROMEDIOS }\end{array}$ & SIGNIFICANCIA \\
\hline $\begin{array}{l}76 \text { países han perdido ya todos sus bosques primarios y otros } 11 \text { estan en vía de } \\
\text { extinción }\end{array}$ & 18.2 & .000 \\
\hline La participación de niños (si la hubiera) no afecta adversamente sus bienes & 16.6 & .000 \\
\hline Los bosques tropicales han perdido $80 \%$ de su superficie & 15.4 & .000 \\
\hline Los tránsgenicos y el glifosofato causan daños a la salud y la deterioran & 14.6 & .000 \\
\hline Los problemas ambientales de Latinoamérica incluyen degradación & 14.5 & .000 \\
\hline Se opone a la mercantilización y las patentes de los bienes comunes & 14.4 & .000 \\
\hline Valora y recompensa debidamente el trabajo de la mujer & 13.7 & .000 \\
\hline Promueve el comercio transparente, que garantizan ingresos dignos para todos & 13.7 & .000 \\
\hline Da prioridad a las economías y a los mercados locales y nacionales & 12.6 & .000 \\
\hline Promueven activamente mejores prácticas medioambientales & 12.3 & .000 \\
\hline Los productores trabajan en un entorno seguro saludable & 12.1 & .000 \\
\hline Definición soberanía alimentaria & 12.1 & .000 \\
\hline Respeta el derecho de alimentación y nutrición de los consumidores & 11.6 & .000 \\
\hline La producción de alimentos se base en la sostenibilidad medioambiental & 11.6 & .000 \\
\hline Se mantienen relaciones basadas en la solidaridad y confianza & 11.4 & .015 \\
\hline El precio justo se acuerda a traves de diálogo y la participación & 11.4 & .000 \\
\hline Las organizaciones de CJ generan conciencia sobre CJ & 11.1 & .000 \\
\hline Siguen estándares más altos en calidad y empaquetado del producto & 10.8 & .000 \\
\hline Permite una producción socialmente justa y ambientalmente responsable & 10.1 & .000 \\
\hline Definición de desarrollo sostenible & 9.8 & .000 \\
\hline Utilizan técnicas honestas de publicidad y mercado & 9.7 & .000 \\
\hline Es una estrategia para aliviar la pobreza y propiciar el desarrollo sostenible & 9.6 & .000 \\
\hline Da oportunidad a productores en desventaja en el comercio convencional & 8.9 & .000 \\
\hline Paga un sobre precio por la misma calidad si hay un valor ético adicional & 8.1 & .010 \\
\hline Consideran dónde y como se ha fabricado el producto & 7 & .000 \\
\hline Mantiene su compromiso con el medioambiente (reduce, recicla, reúsa) & 6.3 & .000 \\
\hline $\begin{array}{l}\text { Está interesado por el origen del producto y a quién se beneficia con su comer- } \\
\text { cio... }\end{array}$ & 5.3 & .000 \\
\hline Esta motivado por su postura ética & 2.6 & .000 \\
\hline Minimiza el consumo y evita el despilfarro & 2.4 & .088 \\
\hline Distingue entre sus necesidades y los deseos generados por la publicidad & 1.7 & .000 \\
\hline
\end{tabular}

Fuente: Elaboración propia. 


\section{Indice de propensidad de adoptar comportamientos}

Los resultados muestran que los promedios más altos se obtuvieron en acciones relacionadas a las etapas de preparación y de acciones individuales. Los más bajos se obtuvieron en acciones de la etapa contemplativa y de acciones colectivas.

Las acciones de la etapa de preparación con puntajes más altos se refieren a acciones de cambios de actitudes personales para ser más responsables.

Las acciones individuales que los participantes tienen más probabilidad de realizar en un futuro inmediato están relacionadas con reciclar, añadir alimentos saludables, reducir el desperdicio de agua. Hacer composta en casa, y boicotear productos y

Tabla No 04:

Propensidad de adoptar comportamientos/índice de propensidad por etapa (Objetivo $\mathrm{N}^{\circ} 4$ )

\begin{tabular}{|c|c|c|}
\hline ACCIONES & Promedio & Des.Est. \\
\hline \multicolumn{3}{|l|}{ ETAPA CONTEMPLATIVA } \\
\hline \multicolumn{3}{|l|}{ Investigar sobre: } \\
\hline Reciclaje y otros temas ambientales & 64.8 & 32.9 \\
\hline Los organismos genéticamente modificados & 64.8 & 32.9 \\
\hline Los materiales o ingredientes de los productos & 65.3 & 31.3 \\
\hline La procedencia de los productos que consumo & 63.4 & 31.9 \\
\hline El proceso de elaboración de los productos & 60.6 & 32.9 \\
\hline Indice Etapa Contemplativa & 63.8 & 32.4 \\
\hline \multicolumn{3}{|l|}{ ETAPA DE PREPARACIÓN } \\
\hline Estar más consciente de las consecuencias & 80.4 & 25.6 \\
\hline No juzgar a las personas por lo que tienen o dejan & 79.3 & 29.4 \\
\hline Distinguir entre necesidades reales y deseos & 76.7 & 26.9 \\
\hline Encontrar nuevos usos a cosas viejas & 72.4 & 28.5 \\
\hline No estar pendiente de las modas & 64.3 & 34.5 \\
\hline Indice Etapa de Preparación & 74.6 & 29.0 \\
\hline \multicolumn{3}{|l|}{ ETAPA DE ACCIONES INDIVIDUALES } \\
\hline Reciclar & 81.0 & 25.6 \\
\hline Añadir alimentos saludables a mi dieta & 82.2 & 24.2 \\
\hline Leer las etiquetas de los alimetos & 76.7 & 27.2 \\
\hline No desechar artículos cuando todavía funcionan & 75.3 & 28.7 \\
\hline Reducir el consumo de agua & 81.9 & 25.9 \\
\hline Comprar productos locales & 79.7 & 23.4 \\
\hline Eliminar o disminuir el consumo de comida chatarra & 75.3 & 28.1 \\
\hline Dejar de comprar y consumir productos tóxicos & 72.9 & 31.2 \\
\hline Reducir el consumo de productos plásticos & 74.5 & 27.6 \\
\hline Dejar de comprar y consumir productos elaborados & 70.0 & 36.6 \\
\hline Usar bolsas de tela para transportar la compra & 76.7 & 27.2 \\
\hline Tomar agua del grifo en vez de botellas plásticas & 68.1 & 35.0 \\
\hline Comprar en mercados agrícolas y cooperativas & 70.7 & 29.9 \\
\hline Reducir el consumo de petroleo y derivados (energía) & 68.4 & 29.8 \\
\hline Moderar mi consumo de aparatos electrónicos & 66.2 & 31.0 \\
\hline Comprar alimentos orgánicos & 66.3 & 32.0 \\
\hline Cultivar algunos de mis alimentos (en el patio, techo, etc) & 54.2 & 39.1 \\
\hline Hacer compost en mi casa & 51.3 & 36.7 \\
\hline Indice Etapa de Acciones Individuales & 65.2 & 32.6 \\
\hline \multicolumn{3}{|l|}{ ETAPA DE ACCIONES COLECTIVAS } \\
\hline Apoyar movimientos civiles pro ambiente & 67.4 & 31.7 \\
\hline Apoyar movimientos civiles pro desarrollo sostenible & 65.7 & 32.4 \\
\hline Dialogar con amigosy familiares sobre consumo & 68.8 & 30.4 \\
\hline Apoyar movimientos civiles pro soberanía alimentaria & 68.8 & 30.4 \\
\hline Apoyar movimientos civiles pro comercio justo & 63.0 & 32.4 \\
\hline Dialogar con amigos y familiares sobre el desarrollo sostenible & 67.4 & 31.4 \\
\hline Dialogar con amigos y familiares sobre la soberanía alimentaria & 64.2 & 32.7 \\
\hline Dialogar con amigos y familiares sobre el comercio justo & 62.4 & 33.4 \\
\hline Aumentar mi participación en asuntos ciudadanos & 58.6 & 32.8 \\
\hline Boicotear productos y compañias no éticas & 48.1 & 36.5 \\
\hline Indice de Acciones Colectivas & 63.5 & 32.4 \\
\hline INDICE DE INTENCIÓN GENERAL & 66.8 & 31.6 \\
\hline
\end{tabular}

Fuente: Elaboración propia.

146/ QVIPURAMAYOC | Vol. 24(46) 2016 
compañías no éticas, son las acciones que los participantes tienen menor intención de realizar.

\section{Indice de Conocimiento y de inquietud ética en las} intenciones de acción por cada etapa de cambio

Se ejecutaron regresiones jerárquicas para las variables dependientes de intenciones de cada etapa y el índice de intención general.

Este método permite que los incrementos en la varianza puedan ser atribuidos a cada serie de variables. (Ver Tablas $\mathrm{N}^{\circ} 05$ y $\mathrm{N}^{\circ} 06$ )

Tabla No 05 y No 6

Efecto del índice del conocimiento y de inquietud ética en las intenciones de acciones de cada etapa de cambio

\begin{tabular}{|c|c|c|c|}
\hline \multicolumn{4}{|c|}{$\begin{array}{l}\text { VARIABLE DEPENDIENTE: Intenciones en Etapa de } \\
\text { Contemplación - Tabla } N^{\circ} 05\end{array}$} \\
\hline & PASO 1 & PASO 2 & PASO 3 \\
\hline \multicolumn{4}{|l|}{$\begin{array}{l}\text { Indíces conoci- } \\
\text { miento Comercio } \\
\text { Justo }\end{array}$} \\
\hline $\begin{array}{l}\text { Indíces conoci- } \\
\text { miento Consumo } \\
\text { Responsable }\end{array}$ & .198 & .158 & .159 \\
\hline $\begin{array}{l}\text { Indíces conoci- } \\
\text { miento Desarrollo } \\
\text { Sostenible }\end{array}$ & .105 & & \\
\hline \multicolumn{4}{|l|}{$\begin{array}{l}\text { Indíces conoci- } \\
\text { miento Soberanía } \\
\text { alimentaria }\end{array}$} \\
\hline \multicolumn{4}{|l|}{$\begin{array}{l}\text { Indíces inquietud } \\
\text { Comercio Justo }\end{array}$} \\
\hline \multicolumn{4}{|l|}{$\begin{array}{l}\text { Indíces inquietud } \\
\text { Consumo Respon- } \\
\text { sable }\end{array}$} \\
\hline $\begin{array}{l}\text { Indíces inquietud } \\
\text { Desarrollo Soste- } \\
\text { nible }\end{array}$ & & .159 & .162 \\
\hline \multicolumn{4}{|l|}{$\begin{array}{l}\text { Indíces inquietud } \\
\text { Soberanía alimen- } \\
\text { taria }\end{array}$} \\
\hline \multicolumn{4}{|l|}{ Sexo } \\
\hline \multicolumn{4}{|l|}{ Edad } \\
\hline \multicolumn{4}{|l|}{$\begin{array}{l}\text { Capacidad Adqui- } \\
\text { sitiva }\end{array}$} \\
\hline$\Delta \mathrm{R}^{2}$ & .084 & .054 & .003 \\
\hline $\mathrm{R}^{2}$ & .084 & .138 & .141 \\
\hline $\mathrm{R}^{2}$ Ajustado & .079 & .128 & .127 \\
\hline
\end{tabular}

Fuente: Elaboración propia.

\begin{tabular}{|c|c|c|c|}
\hline \multicolumn{4}{|c|}{$\begin{array}{l}\text { VARIABLE DEPENDIENTE: Intenciones en Etapa de } \\
\text { Preparación - Tabla } N^{\circ} 06\end{array}$} \\
\hline & PASO 1 & PASO 2 & PASO 3 \\
\hline \multicolumn{4}{|l|}{$\begin{array}{l}\text { Indíces conoci- } \\
\text { miento Comercio } \\
\text { Justo }\end{array}$} \\
\hline $\begin{array}{l}\text { Indíces conoci- } \\
\text { miento Consumo } \\
\text { Responsable }\end{array}$ & .159 & .116 & .117 \\
\hline $\begin{array}{l}\text { Indíces conoci- } \\
\text { miento Desarrollo } \\
\text { Sostenible }\end{array}$ & .168 & .140 & .144 \\
\hline \multicolumn{4}{|l|}{$\begin{array}{l}\text { Indíces conoci- } \\
\text { miento Soberanía } \\
\text { alimentaria }\end{array}$} \\
\hline \multicolumn{4}{|l|}{$\begin{array}{l}\text { Indíces inquietud } \\
\text { Comercio Justo }\end{array}$} \\
\hline \multicolumn{4}{|l|}{$\begin{array}{l}\text { Indíces inquietud } \\
\text { Consumo Respon- } \\
\text { sable }\end{array}$} \\
\hline $\begin{array}{l}\text { Indíces inquietud } \\
\text { Desarrollo Soste- } \\
\text { nible }\end{array}$ & & .216 & \\
\hline $\begin{array}{l}\text { Indíces inquietud } \\
\text { Soberanía alimen- } \\
\text { taria }\end{array}$ & & & .215 \\
\hline \multicolumn{4}{|l|}{ Sexo } \\
\hline \multicolumn{4}{|l|}{ Edad } \\
\hline \multicolumn{4}{|l|}{$\begin{array}{l}\text { Capacidad Adqui- } \\
\text { sitiva }\end{array}$} \\
\hline$\Delta \mathrm{R}^{2}$ & .060 & .083 & .006 \\
\hline $\mathrm{R}^{2}$ & .060 & .143 & .149 \\
\hline $\mathrm{R}^{2}$ Ajustado & .054 & .133 & .136 \\
\hline
\end{tabular}

Fuente: Elaboración propia. 
Los resultados muestran que los índices de conocimiento e inquietud ética sobre el desarrollo sostenible son mucho mejores predictores de las intenciones generales que los índices generales, mejorando significativamente el poder explicativo del modelo. El modelo con más poder explicativo resultó ser el de las intenciones en la etapa de acciones individuales, seguido del de la etapa de preparación, la de contemplación, concluyendo con el de las acciones colectivas. (Ver tablas $\mathrm{N}^{\circ} 07,08$ y 09 )

\section{Tabla No 07 y No 8}

Efecto del índice del conocimiento y de inquietud ética en las intenciones de acciones de cada etapa de cambio

VARIABLE DEPENDIENTE: Intenciones en Etapa de Acciones Individuales - Tabla $\mathbf{N}^{\circ} 07$

\begin{tabular}{|c|c|c|c|}
\hline & PASO 1 & PASO 2 & PASO 3 \\
\hline \multicolumn{4}{|l|}{$\begin{array}{l}\text { Indíces conoci- } \\
\text { miento Comercio } \\
\text { Justo }\end{array}$} \\
\hline $\begin{array}{l}\text { Indíces conoci- } \\
\text { miento Consumo } \\
\text { Responsable }\end{array}$ & .177 & .127 & .129 \\
\hline \multicolumn{4}{|l|}{$\begin{array}{l}\text { Indíces conoci- } \\
\text { miento Desarrollo } \\
\text { Sostenible }\end{array}$} \\
\hline \multicolumn{4}{|l|}{$\begin{array}{l}\text { Indíces conoci- } \\
\text { miento Soberanía } \\
\text { alimentaria }\end{array}$} \\
\hline $\begin{array}{l}\text { Indíces inquietud } \\
\text { Comercio Justo }\end{array}$ & & & .108 \\
\hline \multicolumn{4}{|l|}{$\begin{array}{l}\text { Indíces inquietud } \\
\text { Consumo Respon- } \\
\text { sable }\end{array}$} \\
\hline $\begin{array}{l}\text { Indíces inquietud } \\
\text { Desarrollo Soste- } \\
\text { nible }\end{array}$ & & .242 & .245 \\
\hline \multicolumn{4}{|l|}{$\begin{array}{l}\text { Indíces inquietud } \\
\text { Soberanía alimen- } \\
\text { taria }\end{array}$} \\
\hline Sexo & & & .073 \\
\hline \multicolumn{4}{|l|}{ Edad } \\
\hline \multicolumn{4}{|l|}{$\begin{array}{l}\text { Capacidad Adqui- } \\
\text { sitiva }\end{array}$} \\
\hline$\Delta \mathrm{R}^{2}$ & .067 & .087 & .005 \\
\hline $\mathrm{R}^{2}$ & .067 & .154 & .159 \\
\hline $\mathrm{R}^{2}$ Ajustado & .062 & .144 & .146 \\
\hline
\end{tabular}

Fuente: Elaboración propia.

\begin{tabular}{|c|c|c|c|}
\hline \multicolumn{4}{|c|}{$\begin{array}{l}\text { VARIABLE DEPENDIENTE: Intenciones en Etapa de } \\
\text { de Acciones Colectivas - Tabla } N^{\circ} 08\end{array}$} \\
\hline & PASO 1 & PASO 2 & PASO 3 \\
\hline \multicolumn{4}{|l|}{$\begin{array}{l}\text { Indíces conoci- } \\
\text { miento Comercio } \\
\text { Justo }\end{array}$} \\
\hline \multicolumn{4}{|l|}{$\begin{array}{l}\text { Indíces conoci- } \\
\text { miento Consumo } \\
\text { Responsable }\end{array}$} \\
\hline \multicolumn{4}{|l|}{$\begin{array}{l}\text { Indíces conoci- } \\
\text { miento Desarrollo } \\
\text { Sostenible }\end{array}$} \\
\hline $\begin{array}{l}\text { Indíces conoci- } \\
\text { miento Soberanía } \\
\text { alimentaria }\end{array}$ & .174 & .151 & .165 \\
\hline $\begin{array}{l}\text { Indíces inquietud } \\
\text { Comercio Justo }\end{array}$ & & .124 & .119 \\
\hline \multicolumn{4}{|l|}{$\begin{array}{l}\text { Indíces inquietud } \\
\text { Consumo Respon- } \\
\text { sable }\end{array}$} \\
\hline \multicolumn{4}{|l|}{$\begin{array}{l}\text { Indíces inquietud } \\
\text { Desarrollo Soste- } \\
\text { nible }\end{array}$} \\
\hline \multicolumn{4}{|l|}{$\begin{array}{l}\text { Indíces inquietud } \\
\text { Soberanía alimen- } \\
\text { taria }\end{array}$} \\
\hline \multicolumn{4}{|l|}{ Sexo } \\
\hline \multicolumn{4}{|l|}{ Edad } \\
\hline \multicolumn{4}{|l|}{$\begin{array}{l}\text { Capacidad Adqui- } \\
\text { sitiva }\end{array}$} \\
\hline$\Delta \mathrm{R}^{2}$ & .064 & .031 & .008 \\
\hline $\mathrm{R}^{2}$ & .064 & .095 & .103 \\
\hline $\mathrm{R}^{2}$ Ajustado & .058 & .084 & .088 \\
\hline
\end{tabular}

Fuente: Elaboración propia. 
Tabla No 9

Efecto del índice del conocimiento y de inquietud ética en las intenciones de acciones de cada etapa de cambio

\begin{tabular}{|c|c|c|c|}
\hline \multicolumn{4}{|c|}{$\begin{array}{l}\text { VARIABLE DEPENDIENTE: Intenciones Generales - } \\
\text { Tabla } \text { N}^{\circ} 09\end{array}$} \\
\hline & PASO 1 & PASO 2 & PASO 3 \\
\hline \multicolumn{4}{|l|}{$\begin{array}{l}\text { Indíces conocimien- } \\
\text { to Comercio Justo }\end{array}$} \\
\hline $\begin{array}{l}\text { Indíces conoci- } \\
\text { miento Consumo } \\
\text { Responsable }\end{array}$ & .176 & .132 & .133 \\
\hline \multicolumn{4}{|l|}{$\begin{array}{l}\text { Indíces conoci- } \\
\text { miento Desarrollo } \\
\text { Sostenible }\end{array}$} \\
\hline $\begin{array}{l}\text { Indíces conoci- } \\
\text { miento Soberanía } \\
\text { alimentaria }\end{array}$ & .151 & .126 & .131 \\
\hline $\begin{array}{l}\text { Indíces inquietud } \\
\text { Comercio Justo }\end{array}$ & & & .115 \\
\hline \multicolumn{4}{|l|}{$\begin{array}{l}\text { Indíces inquietud } \\
\text { Consumo Respon- } \\
\text { sable }\end{array}$} \\
\hline $\begin{array}{l}\text { Indíces inquietud } \\
\text { Desarrollo Sostenible }\end{array}$ & & & .193 \\
\hline \multicolumn{4}{|l|}{$\begin{array}{l}\text { Indíces inquietud } \\
\text { Soberanía alimentaria }\end{array}$} \\
\hline \multicolumn{4}{|l|}{ Sexo } \\
\hline \multicolumn{4}{|l|}{ Edad } \\
\hline \multicolumn{4}{|l|}{$\begin{array}{l}\text { Capacidad Adqui- } \\
\text { sitiva }\end{array}$} \\
\hline$\Delta \mathrm{R}^{2}$ & .087 & .077 & .004 \\
\hline $\mathrm{R}^{2}$ & .087 & .164 & .168 \\
\hline $\mathrm{R}^{2}$ Ajustado & .082 & .154 & .154 \\
\hline
\end{tabular}

Fuente: Elaboración propia.

\section{HIPÓTESIS PLANTEADAS}

Primera Hipótesis: El índice de conocimiento de un conflicto ético está positivamente correlacionado a la propensidad de adoptar comportamientos éticos orientados al desarrollo sostenible en cada una de las etapas de cambio.

Los resultados de cuatro de los cinco modelos de regresión proveen evidencia sobre la correlación entre el índice de conocimiento, sobre el desarrollo sostenible y la intención de actuar en cada una de las etapas, excepto en la de preparación, que se correla- ciona con el índice de conocimiento sobre consumo responsable. Las variables independientes de cada modelo tienen coeficientes betas estandarizados positivos y significativos, excepto en la etapa de preparación, que hay un coeficiente negativo. Estos hallazgos proveen evidencia de apoyo a la primera hipótesis sugiriendo que mientras más conocimiento tenga una persona sobre un conflicto ético, más alta será la probabilidad de que adopte un comportamiento ético al respecto, o viceversa. No obstante, 
contrario a lo esperado, la correlación entre el nivel de conocimiento y la intención de actuar desaparece por completo cuando se entra al modelo la variable de la inquietud ética, (excepto en la etapa de preparación), lo que sugiere, que el conocimiento se convierte en inquietud ética creando una correlación más fuerte con la intención de actuar éticamente. Contrario a la primera hipótesis, los índices de conocimiento sobre el comercio justo y la soberanía alimentaria no tuvieron correlación significativa con la variable dependiente en ninguno de los modelos.

Segunda Hipótesis: El índice de inquietud ética está positivamente correlacionado con la propensidad de adoptar comportamientos éticos orientados al desarrollo sostenible en cada una de las etapas de cambio.

En el segundo paso de las regresiones jerárquicas se añadieron los índices de inquietud ética, para determinar su impacto en la intención de adoptar comportamientos éticos, orientados al desarrollo sostenible en cada una de las etapas de cambio. Este paso resultó en un aumento significativo de los coeficientes de determinación, ajustados en todos los casos proveyendo apoyo a la segunda hipótesis. Los coeficientes ajustados beta sugieren que los individuos tienden a actuar éticamente, para resolver una inquietud ética que provoca un estado desagradable de tensión moral, según lo propuesto en el modelo de toma de decisiones aquí examinado. Es importante notar, sin embargo, que solo el índice de inquietud el sobre desarrollo sostenible tuvo correlaciones significativas en todos los modelos. Los índices de inquietud sobre los otros dos temas no fueron significativos en ningún modelo.

Tercera Hipótesis: El sexo, la capacidad adquisitiva y la edad de los participantes están correlacionados con la propensidad de adoptar comportamientos éticos orientados al desarrollo sostenible en cada una de las etapas de cambio.

En el tercer paso de las regresiones jerárquicas, se añadieron las tres variables personales para determinar su impacto en la intención de adoptar comportamientos éticos, orientados al desarrollo sostenible en cada una de las etapas de cambio. Este paso resultó en un aumento ligero de los coeficientes de determinación, ajustados en todos los casos, pero ninguna de las variables examinadas resultó con coeficientes Beta significativos. Por lo tanto, los resultados no proveen evidencia de apoyo a esta hipótesis. Este resultado contradice los estudios previos en otros países, que hallaron que las mujeres mileniales son más propensas a convertirse en consumidoras responsables y apoyar a los movimientos sociales afines con el desarrollo sostenible.

\section{ANÁLISIS DE LOS RESULTADOS}

Los resultados apoyan parcialmente las primeras dos hipótesis, pero nola tercera. La primera hipótesis plantea una relación entre el nivel de conocimiento de un conflicto ético y la intención de adoptar comportamientos éticos y la segunda lo propio, con el índice de inquietud ética. Los resultados apoyan el planteamiento principal del modelo teórico, aquí propuesto, de que el individuo no tendrá intención de actuar éticamente, si el conocimiento adquirido sobre un posible conflicto no se convierte en una inquietud ética, definida como un estado desagradable de tensión moral. A raíz de los hallazgos de este estudio se puede argumentar que el conocimiento sobre un conflicto ético es un antecedente necesario para crear la suficiente disonancia moral que provoque la intención de cambiar el comportamiento. Esto provee evidencia de que la sustentación teórica para integrar la teoría de la disonancia cognitiva de Festinger (1957) al modelo de toma de decisiones de Rest (1986) es adecuada y relevante en la actualidad.

Tomados en conjunto los resultados de este estudio, sugieren que los participantes tienen un conocimiento bastante limitado sobre el desarrollo sostenible y marcadamente deficiente, de los movimientos sociales asociados a éste: comercio justo, soberanía alimentaria y consumo responsable. Por ello, parece ser que los participantes están comenzando a convertir el conocimiento sobre el desarrollo sostenible en inquietud ética, resultando que las intenciones de adoptar comportamientos más acordes con el desarrollo sostenible, están entre las etapas de preparación y la de acciones individuales. 
En cuanto a los demás conflictos, parecen estar más, en una etapa pre contemplativa, en la que todavía no tienen el conocimiento y mucho menos la inquietud ética para considerar cambios de hábitos. De igual forma, los resultados sugieren que el grado de conocimiento e inquietud ética que tienen actualmente sobre los temas, tampoco los sitúa en la última etapa del cambio, cuando se coordinan y concatenan acciones propias con las del colectivo; ejerciendo así, sus prerrogativas ciudadanas. Estos resultados apuntan a la deseabilidad de que las escuelas de negocios reevalúen su función formadora, para incluir en sus modelos educativos temas éticos primordiales y urgentes, que los contadores y administradores del siglo 21 deben no solo conocer, sino contribuir a su resolución.

\section{LOGRO DE OBJETIVOS}

Como producto de la aplicación de la base de datos, el análisis de sus resultados y considerando los alcances de la contemplación que involucra: la preparación, las acciones individuales, las acciones colectivas, los índices de conocimiento de la Inquietud Ética. En base a los temas de estudio, los conceptos materia de investigación, en concordancia con los resultados obtenidos y finalmente, la situación del tema propuesto; se reflejaría en los resultados de la aplicación estadística cuando se responda en el análisis de las variables dependientes e independientes.

Este estudio logró los cinco objetivos propuestos. Primero, se calcularon los Índices de conocimiento de los participantes sobre el desarrollo sostenible, el comercio justo, el consumo responsable y la soberanía alimentaria, reflejando un conocimiento bastante escaso de los temas.

Segundo, se calcularon los Índices de Inquietud Ética sobre los temas tratados, encontrando que los participantes tienen una inquietud ética moderada sobre el desarrollo sostenible y muy débil, respecto a los demás asuntos.

Tercero, se identificaron diferencias significativas entre el Índice de Conocimiento y de Inquietud Ética de los participantes, diferenciando la reacción cognitiva de la afectiva.
Cuarto, se determinó la propensidad de adoptar comportamientos característicos de las diferentes fases del modelo de etapas de cambio y calcular un índice de propensidad para cada etapa, encontrando que los participantes están entre las etapas de preparación y acciones individuales en cuanto al desarrollo sostenible, y en la etapa contemplativa en los otros asuntos éticos abordados en este estudio.

Quinto, este estudio propuso y examinó un modelo de toma de decisiones, en el que se integraron elementos de la teoría de la Disonancia Cognoscitiva y el modelo Transteórico de las Etapas de Cambio. Encontrándose evidencia de apoyo en dos de las tres hipótesis propuestas. Este estudio identificó que el conocimiento es un antecedente necesario, pero no suficiente para lograr cambios de comportamientos. Es necesario que el conocimiento se convierta en una inquietud ética, para que la intención de cambio surja $y$ avance en las diferentes etapas del cambio del comportamiento, hasta lograr el cambio en la persona.

\section{CONCLUSIONES RECOMENDACIONES}

El estudio, así como la metodología propuesta para nuestro análisis, es positiva, en cuanto al desarrollo las teorías y métodos hasta acá aplicados, en relación a la inquietud ética y sus movimientos; sin embargo, tienen limitaciones en las muestras que no fueron seleccionadas aleatoriamente, no siendo por ello absolutamente confiable.

Las metodologías aplicadas en la fecha, son susceptibles de ser mejoradas incluyendo variables que incrementen el conocimiento de los temas que materia de investigación, dado que en sus resultados se observa la necesidad de seguir investigando, por el interés general que corresponde a los consumidores latinoamericanos y del mundo, a fin de preservar el desarrollo sustentable de los países.

Las facultades de negocios deben promover, que las curriculas de estudio deban estar integradas por lo menos con dos materias sobre los temas analizados, para que se tenga conciencia de tener en cuenta, en su actuar los principios éticos, de comercio justo, consumo responsable y soberanía alimenticia y mejorar la sostenibilidad del medio ambiente. 
El grupo de docentes acordó seguir con la investigación incluyendo una encuesta dirigida a ver los efectos de la política como un agregado en las investigaciones.

\section{REFERENCIAS BIBLIOGRÁFICAS}

1. ARTEAGA, F., FAYOS, T., GALLARZA, M. Y SERVERA, D.(2012) La motivación de compra de productos de comercio justo: propuesta de un índice de medición por diferencias sociodemográficas. Análisis y estudio del consumo responsable y el comercio con justicia como formas de cooperación internacional al desarrollo. España, Universidad Católica de Valencia.

2. Asociación de campesinos y campesinas (1996). Concepto de Soberanía Alimentaria. La Cumbre Mundial sobre Alimentación.

3. BUENDÍA, I., COQUE, J. Y GARCÍA, J. (2001). Comercio justo. La ética en las relaciones comerciales dentro de un entorno globalizado. Distribución y Consumo, 56 (febrero-marzo), 23-33.

4. FESTINGER, L (1975) Teoría de la disonancia cognoscitiva. Madrid, Instituto de Estudios Políticos.
5. GIGLIOTTI, M. (2009). Conoscenza e comportamento di acquisto dei prodotti equosolidali negli studenti universitari italiani. 8th International Congress Marketing Trends, París, enero 1617, 2009.

6. LÓPEZ, S. Y RIVERA, B. (2016) Modelo de toma de decisiones éticas. Guía de Investigación Cuantitativa para Explorar: El Razonamiento Bioético y la Orientación hacia el Desarrollo Sostenible y sus Movimientos Afines. Asociación Latinoamericana de Facultades y Escuelas de Contaduría y Administración (ALAFEC) y su Red de Doctorados. Universidad de Texas Rio Grande Valley. Texas, Estados Unidos.

7. PROCHASKA, J. Y DICLEMENTE, C.(1984). The transtheoritical approach: Crossing the traditional boundaries of therapy. Homewood. Illinois: Dorsey Press

8. REST, J. (1986). Moral development. Advances in research and theory. New York;Praeger.

9. ROMAN, S. Y MUNERA, J. (2005). Determinants and consequences of ethical behavior: An empirical study of sales people. European Journal of Marketing, 5/6 (3), 473-495 